\title{
Avaliação de padrão irregular dos escudos do casco em Podocnemis expansa e Podocnemis unifilis (Testudines, Podocnemididae)
}

\author{
Giovanni SALERA-JUNIOR ${ }^{1}$, Adriana MALVASIO ${ }^{2}$, Thiago Costa Gonçalves PORTELINHA ${ }^{3}$
}

\section{RESUMO}

O casco de Podocnemis expansa (tartaruga-da-amazônia) e Podocnemis unifilis (tracajá), os maiores quelônios de água doce da América do Sul, é uma estrutura única que diferencia esse grupo dos vertebrados atuais e está associado a alguns padrōes comportamentais. O padrão dos escudos que compõem o casco dos quelônios é bastante uniforme, mas variações já foram descritas e analisadas para um grande número de espécies em todas as famílias existentes atualmente. Averiguou-se a incidência de irregularidades do padrão de escutelação no casco dessas espécies, durante os anos de 1999 a 2003 no rio Javaés, entorno do Parque Nacional do Araguaia e da Área de Proteção Ambiental Bananal/ Cantão, oeste do estado do Tocantins. P. expansa se apresenta como uma espécie mais suscetível à ocorrência de anormalidades no casco em relação a $P$. unifilis. Sendo que, dos 14.378 filhotes avaliados, (13,32 \%) apresentavam irregularidades no padrão de escutelação enquanto que dos 1.329 filhotes de $P$. unifilis apenas (4,44\%). Em P. expansa, os defeitos ocorreram quase que em sua totalidade na carapaça (98,64\%) e em menor número $(83,05 \%)$ em $P$. uniflis.

PALAVRAS-CHAVE: Podocnemis expansa, Podocnemis unifilis, anormalidade, casco, rio Javaés.

\section{Evaluation of irregular pattern of the shell scutes in Podocnemis expansa and Podocnemis unifilis (Testudines, Podocnemididae)}

\section{ABSTRACT}

The shell of Podocnemis expansa (Amazonian giant River turtle) and Podocnemis unifilis (Yellow-spotted Amazon River Turtle), the largest South American turtles, is a unique structure that differentiates them from other known vertebrates; and it is associated with some of their behavioral patterns. Their scute pattern is quite uniform however variations have been described and analyzed for a great number of other species of existing families. In the Javaés River, from 1999 to 2003, we observed an occurrence of abnormalities in their scute pattern. This was in the vicinity of the Araguaia National Park and the Bananal/ Cantão Environment Protected Area, west of Tocantins state. Of the both species, $P$. expansa is more susceptible to shell abnormalities. Of 14.378 P. expansa hatchlings observed, $13.32 \%$ presented scute pattern abnormalities, while of $1.329 P$. unifilis hatchlings, only $4.44 \%$. The incidence of abnormalities occurred only in the carapace, namely, $98.64 \%$ in $P$. expansa, and $83.05 \%$ in $P$. unifilis.

KEY WORDS: Podocnemis expansa, Podocnemis unifilis, abnormal shell, Javaés River.

1 Universidade Federal do Tocantins - salerajunior@yahoo.com.br

2 Universidade Federal do Tocantins - malvasio@uft.edu.br

${ }^{3}$ Universidade de São Paulo, Escola Superior de Agricultura "Luiz de Queiroz" - thiagoportelinha@yahoo.com.br 


\section{INTRODUÇÃO}

O casco dos Testudines é uma estrutura única que diferencia esse grupo dos demais vertebrados (Pough et al., 1993), estando intimamente associado a alguns padrōes comportamentais (Molina, 1992). A porção dorsal convexa é a carapaça e a parte ventral mais achatada é o plastrão. As vértebras torácicas e as costelas estão geralmente fundidas com a carapaça óssea (Storer et al., 1989). Os ossos da carapaça geralmente são recobertos por escudos córneos de origem epidérmica que não coincidem, em número e posição com os ossos subjacentes, tornando assim essa estrutura extremamente resistente a choques mecânicos (Pough et al., 1993).

A forma, tamanho, coloração, número e disposição dos escudos que compõem o casco são características importantes na identificação genérica e específica dos Testudines (Medem, 1976; Pritchard \& Trebbau, 1984; Molina \& Rocha, 1996). O padrão dos escudos que compõem o casco dos quelônios da mesma espécie é bastante uniforme (Pritchard, 1979), mas variações nesse padrão já foram descritas e analisadas para um grande número de espécies em todas as famílias existentes atualmente (Zangerl \& Johnson, 1957). Existem diversas possíveis causas da ocorrência desses defeitos entre os quelônios: o manuseio dos ovos (Mast \& Carr, 1989), exposição dos ovos à baixa umidade (Lynn \& Ullrich, 1950), a baixas temperaturas (Molina, 1995) e elevadas temperaturas durante a incubação (Mallmann, 1994).

Podocnemis expansa (tartaruga-da-amazônia) e Podocnemis unifilis (tracajá) são os maiores quelônios de água doce da América do Sul, distribuindo-se pelas bacias Amazônica e do Orinoco, onde apresentam grande importância econômica, tendo sua carne e ovos utilizados como fonte alimentar alternativa para diferentes grupos de ribeirinhos, pescadores e indígenas. O padrão de arranjo e disposição dos escudos do casco dessas duas espécies apresenta-se semelhante, sendo o casco formado por 37 escudos na carapaça e 13 escudos no plastrão (IBAMA, 1989a).

Apesar dessas espécies serem protegidas no Brasil há mais de 25 anos em dezenas de áreas de sua distribuição e já existirem desde 1992 inúmeros criatórios com fins comerciais, ainda perdura uma escassez de informação sobre muitos aspectos relacionados à biologia reprodutiva. Atualmente, não existem relatos na literatura sobre estudos enfocando a ocorrência de irregularidades no padrão de escutelação dessas espécies. Portanto, este trabalho teve por objetivo quantificar e qualificar a ocorrência de irregularidades no padrão de escudos que compõem o casco de $P$. expansa e $P$. unifilis.

\section{MATERIAIS E MÉTODOS}

O estudo foi realizado no rio Javaés, oeste do Estado do Tocantins, no entorno do Parque Nacional do Araguaia e da Área de Proteção Ambiental Bananal/ Cantão, durante cinco anos (1999-2003), nos meses de outubro e novembro. Neste local, o Centro de Conservação e Manejo de Répteis e Anfíbios do Instituto Chico Mendes de Conservação da Biodiversidade (RAN/ ICMBio) coordena atividades do Projeto Quelônios da Amazônia (Figura 1).

Foram observadas as irregularidades no padrão dos escudos em 14.378 filhotes recém-eclodidos de P. expansa, e 1.329 de P. unifilis provenientes de ninhos naturais de cinco praias (Canguçu, Comprida, Coco, Goiaba e Bonita) da margem direita do rio Javaés (Tabelas 1 e 2). Quando observadas, as irregularidades presentes no casco eram desenhadas e descritas.

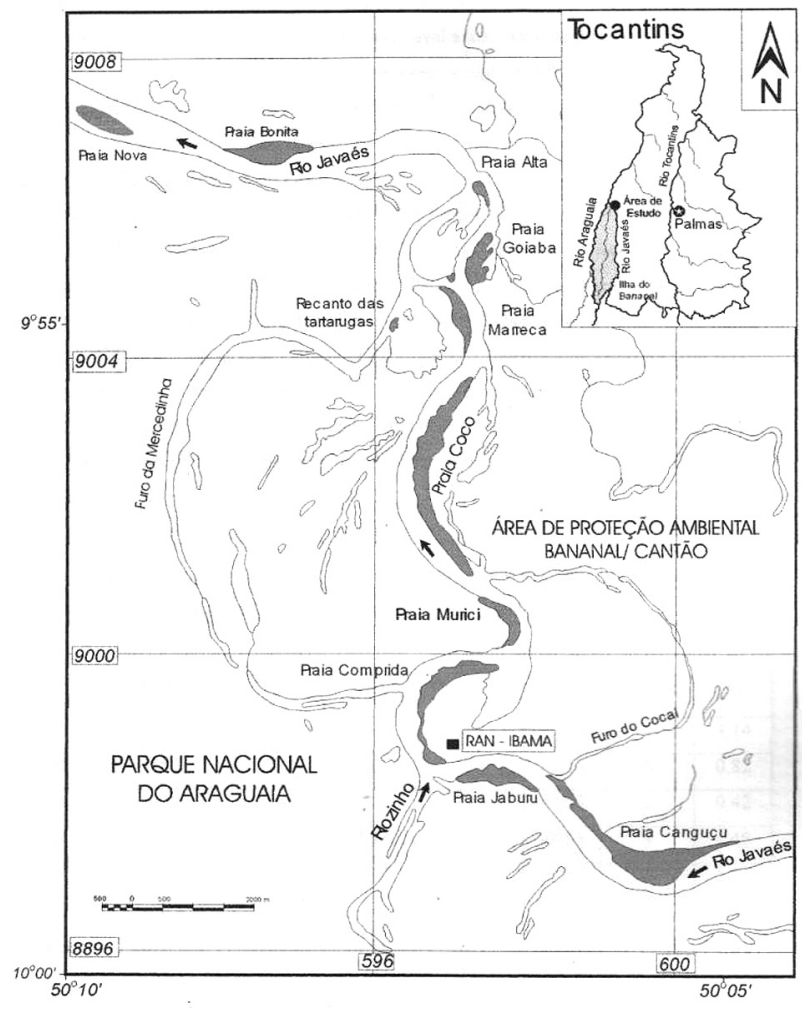

Figura 1 - Localização da área de estudo. Fonte: Ferreira Jr. (2003). 
Tabela 1 - Número de covas e filhotes de Podocnemis expansa avaliados no rio Javaés, Tocantins.

\begin{tabular}{ccc}
\hline Ano & Covas & Filhotes \\
\hline 1999 & 12 & 1.024 \\
2000 & 55 & 5.655 \\
2001 & 19 & 1.740 \\
2002 & 44 & 3.925 \\
2003 & 25 & 2.034 \\
\hline Total & 155 & 14.378 \\
\hline
\end{tabular}

Tabela 2 - Número de covas e filhotes de Podocnemis unifilis avaliados no rio Javaés, Tocantins.

\begin{tabular}{ccc}
\hline Ano & Covas & Filhotes \\
\hline 1999 & 5 & 49 \\
2000 & 36 & 476 \\
2001 & 41 & 463 \\
2002 & 10 & 116 \\
2003 & 23 & 225 \\
\hline Total & 115 & 1.329 \\
\hline
\end{tabular}

\section{RESULTADOS}

Dos 14.378 filhotes de $P$. expansa avaliados, verificamos que $1.916(13,32 \%)$ apresentavam padrão irregular dos escudos do casco e que destes, $1.890(98,64 \%)$ localizavam-se na carapaça e apenas $26(1,36 \%)$ apresentaram-se no plastrão (Tabela 3).

Dos 1.329 filhotes de $P$. unifilis avaliados, verificamos que $59(4,44 \%)$ apresentavam padrão irregular dos escudos do casco e que destes, $49(83,05 \%)$ localizavam-se na carapaça e apenas $10(16,95 \%)$ apresentaram-se no plastrão (Tabela 4).

A Tabela 5, mostra que para P. expansa os 7 defeitos que ocorreram com repetiçôes corresponderam a um total de 1.510 exemplares $(78,82 \%)$, enquanto que os outros defeitos que não ocorreram com repetiçōes corresponderam a 406 exemplares $(21,18 \%)$.

A Tabela 6, mostra que para P.unifilis os 7 defeitos que ocorreram com repetiçóes corresponderam a um total de 18 exemplares $(30,51 \%)$, enquanto que os outros defeitos que não ocorreram com repetiçôes corresponderam a maioria com 41 exemplares $(69,49 \%)$.

Dos 7 principais defeitos registrados, 5 ocorreram na região posterior da carapaça e 2 na região gular do plastrão (Figuras 2-8).
Quando algum filhote apresentava dois ou mais dentre os 7 defeitos observados com repetições, esses diferentes defeitos eram contabilizados como outro defeito.

Desses 7 defeitos registrados com repetiçóes, 2 referem-se ao excesso de escudos vertebrais (Figuras 2 e 5 ), 2 ao excesso de escudos marginais ou supracaudais (Figuras 4 e 6), 1 a

Tabela 3 - Dados do número de filhotes avaliados com padrão irregular de escudos no casco em Podocnemis expansa, no rio Javaés, Tocantins.

\begin{tabular}{|c|c|c|c|c|c|c|c|}
\hline \multirow[b]{2}{*}{ Ano } & \multirow{2}{*}{$\begin{array}{l}\text { Número } \\
\text { de filhotes }\end{array}$} & \multicolumn{6}{|c|}{ Defeitos } \\
\hline & & Total & $(\%)$ & Carapaça & (\%) & Plastrão & $(\%)$ \\
\hline 1999 & 1.024 & 145 & 14,16 & 139 & 95,86 & 6 & 4,14 \\
\hline 2000 & 5.655 & 739 & 13,06 & 733 & 99,18 & 6 & 0,82 \\
\hline 2001 & 1.740 & 241 & 13,85 & 240 & 99,58 & 1 & 0,42 \\
\hline 2002 & 3.925 & 554 & 14,11 & 546 & 98,55 & 8 & 1,45 \\
\hline 2003 & 2.034 & 237 & 11,65 & 232 & 97,89 & 5 & 2,11 \\
\hline Total & 14.378 & 1.916 & - & 1890 & - & 26 & - \\
\hline Média & $2.875,60$ & 383,2 & 13,32 & 378 & 98,64 & 5,2 & 1,36 \\
\hline
\end{tabular}

Tabela 4 - Dados do número de filhotes avaliados com padrão anormal de escudos no casco em Podocnemis unifilis, no rio Javaés, Tocantins.

\begin{tabular}{|c|c|c|c|c|c|c|c|}
\hline \multirow[b]{2}{*}{ Ano } & \multirow[b]{2}{*}{$\begin{array}{l}\text { Número de } \\
\text { filhotes }\end{array}$} & \multicolumn{6}{|c|}{ Defeitos } \\
\hline & & Total & $\%$ & Carapaça & $\%$ & Plastrão & $\%$ \\
\hline 1999 & 49 & 0 & 0 & 0 & 0 & 0 & 0 \\
\hline 2000 & 476 & 23 & 4,83 & 18 & 78,26 & 5 & 21,73 \\
\hline 2001 & 463 & 25 & 5,39 & 21 & 84 & 4 & 16 \\
\hline 2002 & 116 & 3 & 2,58 & 3 & 100 & 0 & 0 \\
\hline 2003 & 225 & 8 & 3,55 & 7 & 87,5 & 1 & 12,5 \\
\hline Total & 1.329 & 59 & - & 49 & - & 10 & - \\
\hline Média & 265,8 & 11,8 & 4,44 & 9,8 & 83,05 & 2 & 16,95 \\
\hline
\end{tabular}

presença de suturas incompletas (Figura 3) e 2 a supressão de escudos no plastrão (Figuras 7 e 8).

Os demais defeitos sem repetições tratam de anormalidades, principalmente, envolvendo excesso de escudos costais e vertebrais.

As únicas anomalias registradas para os filhotes observados tratam-se de irregularidades no padrão dos escudos do casco. Outras anomalias na cabeça, cauda e patas não foram encontradas. 
Tabela 5 - Principais defeitos registrados para Podocnemis expansa, no rio Javaés, Tocantins.

\begin{tabular}{|c|c|c|c|c|c|c|c|c|c|c|}
\hline \multirow{2}{*}{ Ano } & \multirow{2}{*}{ Filhotes } & \multirow{2}{*}{ Defeituosos } & \multicolumn{7}{|c|}{ Defeitos com repetições * } & \multirow{2}{*}{ Outros } \\
\hline & & & Fig. 2 & Fig. 3 & Fig. 4 & Fig. 5 & Fig. 6 & Fig. 7 & Fig. 8 & \\
\hline 1999 & 1024 & 145 & 71 & 33 & 3 & 4 & 4 & 3 & 3 & 24 \\
\hline 2000 & 5.655 & 739 & 315 & 166 & 71 & 47 & 22 & 5 & 1 & 122 \\
\hline 2001 & 1.740 & 241 & 101 & 71 & 8 & 15 & 8 & 1 & 0 & 37 \\
\hline 2002 & 3.925 & 554 & 198 & 112 & 35 & 23 & 15 & 6 & 4 & 161 \\
\hline 2003 & 2034 & 237 & 85 & 53 & 18 & 9 & 6 & 2 & 2 & 62 \\
\hline Total & 14.378 & 1916 & 770 & 436 & 136 & 106 & 57 & 18 & 12 & $406(21,18 \%)$ \\
\hline
\end{tabular}

* 0 s defeitos com repetições estão apresentados nas Figuras 2 à 8 . Mais de um defeito em um mesmo filhote foi contabilizado como outros.

Tabela 6 - Principais defeitos registrados para Podocnemis unifilis, no rio Javaés, Tocantins.

\begin{tabular}{|c|c|c|c|c|c|c|c|c|c|c|}
\hline \multirow{2}{*}{ Ano } & \multirow{2}{*}{ Filhotes } & \multirow{2}{*}{ Defeituosos } & \multicolumn{7}{|c|}{ Defeitos com repetições * } & \multirow{2}{*}{ - Outros } \\
\hline & & & Fig. 2 & Fig. 3 & Fig. 4 & Fig. 5 & Fig. 6 & Fig. 7 & Fig. 8 & \\
\hline 1999 & 49 & 0 & 0 & 0 & 0 & 0 & 0 & 0 & 0 & 0 \\
\hline 2000 & 476 & 23 & 0 & 0 & 0 & 2 & 0 & 2 & 3 & 16 \\
\hline 2001 & 463 & 25 & 0 & 1 & 0 & 1 & 0 & 3 & 1 & 19 \\
\hline 2002 & 116 & 3 & 0 & 0 & 0 & 0 & 0 & 0 & 0 & 3 \\
\hline 2003 & 225 & 8 & 1 & 0 & 1 & 0 & 1 & 2 & 0 & 3 \\
\hline Total & 1329 & 59 & 1 & 1 & 1 & 3 & 1 & 7 & 4 & $41(69,49 \%)$ \\
\hline
\end{tabular}

* 0 s defeitos com repetições estão apresentados nas Figuras 2 à 8 . Mais de um defeito em um mesmo filhote foi contabilizado como outros.

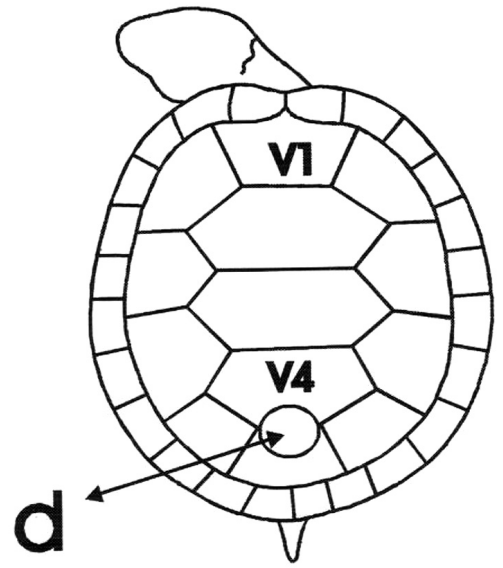

Figura 2 - Deformação no filhote: um escudo a mais entre o quarto e o quinto escudo vertebral: $d$ - defeito, v1 - primeiro escudo vertebral, v4 - quarto escudo vertebral.

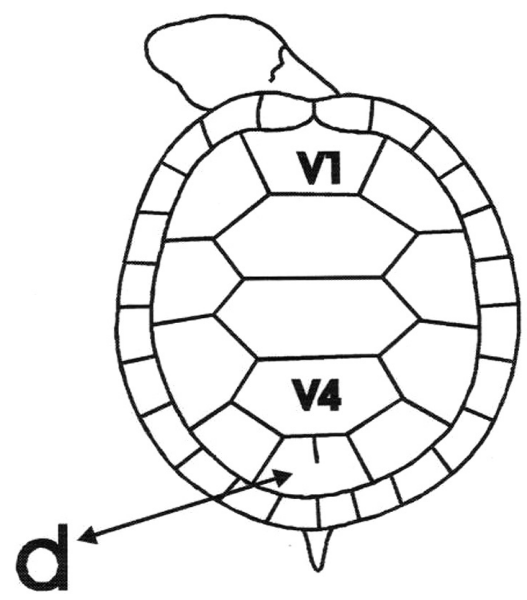

Figura 3 - Deformação no filhote: uma sutura incompleta no quinto escudo vertebral: d- defeito, v1 - primeiro escudo vertebral, v4 - quarto escudo vertebral. 


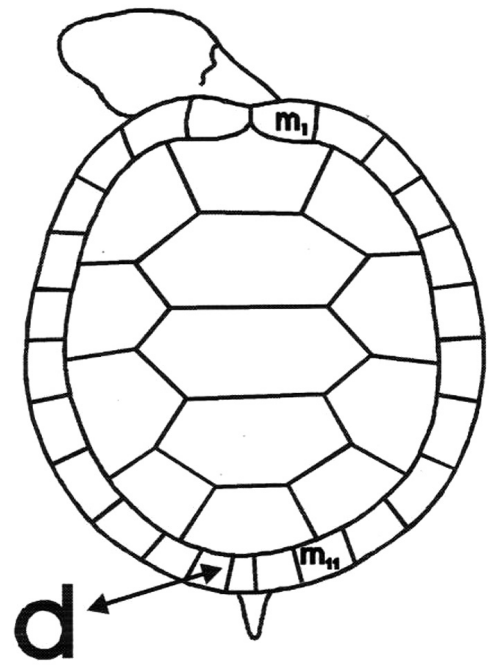

Figura 4 - Deformação no filhote: um escudo marginal ou supracaudal a mais: d -defeito, $\mathrm{m} 1$ - primeiro escudo marginal, $\mathrm{m} 11$ - décimo primeiro escudo marginal.

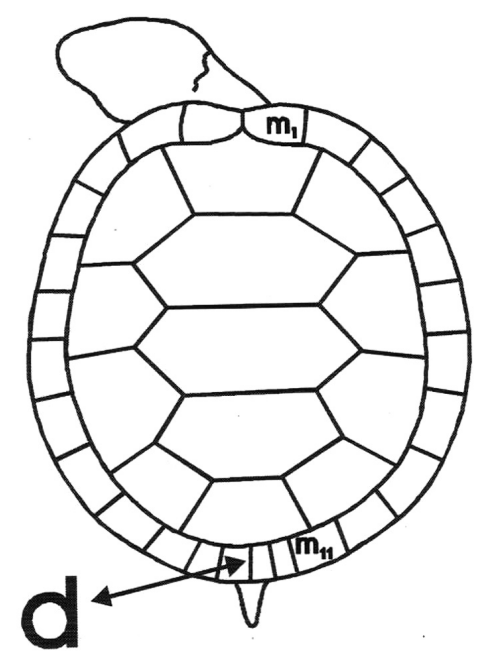

Figura 6 - Deformação no filhote: um par de escudos marginais ou supracaudais a mais: $\mathrm{d}$ - defeito, $\mathrm{m} 1$ - primeiro escudo marginal, $\mathrm{m} 11$ - décimo primeiro escudo marginal.

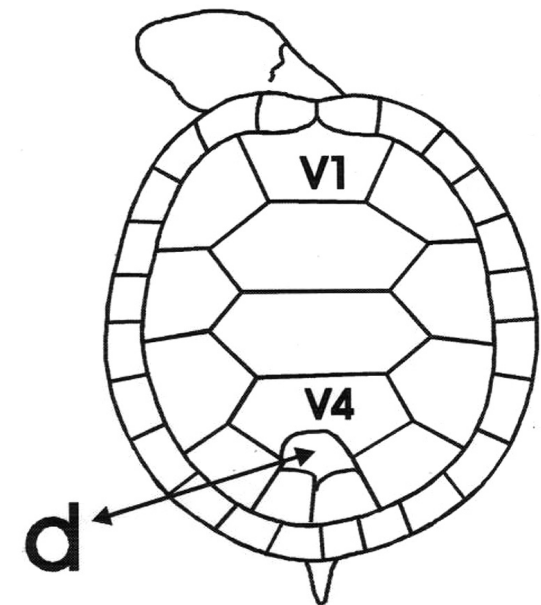

Figura 5 - Deformação no filhote: quinto escudo vertebral dividido em três partes: d - defeito, v1 - primeiro escudo vertebral, v4 - quarto escudo vertebral.

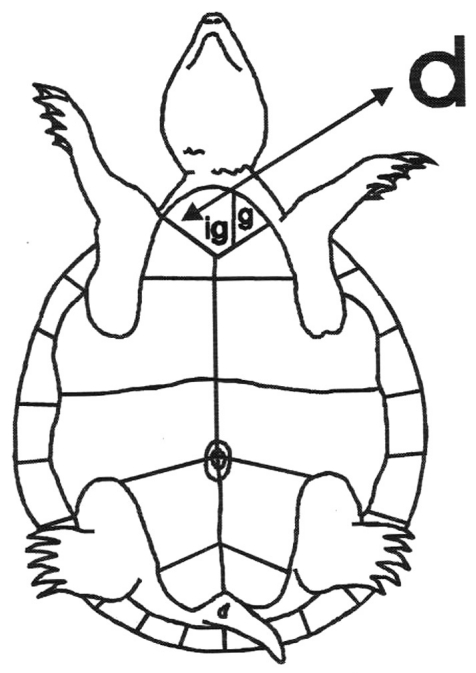

Figura 7 - Deformação no filhote: ausência de sutura unindo o escudo intergular a um escudo gular: $\mathrm{d}$ - defeito, $\mathrm{g}$ - escudo gular, ig - escudo intergular. 


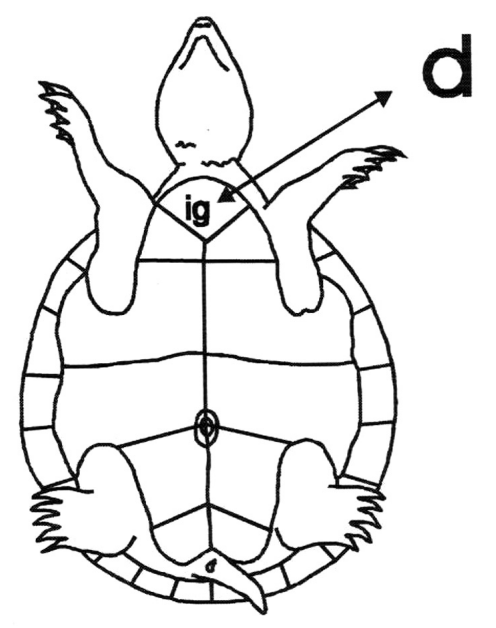

Figura 8 - Deformação no filhote: ausência das suturas unindo o escudo intergular aos escudos gulares: $\mathrm{d}$ - defeito, ig - escudo intergular.

\section{DISCUSSÃO}

Os defeitos no padrão dos escudos do casco de quelônios foram classificados por Zangerl \& Johnson (1957) em três grupos: a) suturas incompletas; b) adição ou supressão de escudos e c) anormalidades envolvendo uma área maior. Esses três grupos de defeitos foram observados nos filhotes de $P$. expansa e $P$. unifilis avaliados, sendo que apenas para os grupos "a" e "b" foram encontradas repetiçôes.

Ewert (1979) complementa a classificação de defeitos apresentando o registro de irregularidades em ziguezague para Sternotherus adoratus, Kinosternon baurii, Chelydra serpentina, Chrysemys picta picta e Chrysemys picta dorsalis (quelônios de água doce) e Molina (1995) para Trachemys dorbignyi (tigre d'água). No presente estudo, também observamos a ocorrência dessas irregularidades em ziguezague para $P$. expansa e $P$. unifilis.

Para Pritchard (1979), os escudos presentes no plastrão são menos suscetíveis à ocorrência de anormalidades, fato observado para Phrynops geoffroanus (cágado) (Molina, 1989), Chelonoidis carbonaria (jabuti) (Andrade \& Abe, 1993; Mallmann, 1994), T. dorbignyi (Molina, 1995), P. expansa e P. unifilis (Malvasio, 2001). Nos indivíduos avaliados, foi observado que os defeitos também ocorreram com maior freqüência na carapaça em relação ao plastrão com valores de $98,64 \%$ e $83,05 \%$ para $P$. expansa e P. unifilis, respectivamente.

Medem (1969), na região do alto rio Caquetá e rio Caguan (Venezuela), observou 136 exemplares jovens e 15 adultos de P. expansa, onde registrou a ocorrência de irregularidades no padrão dos escudos do casco de 8 jovens $(5,88 \%)$ e apenas 1 adulto (6,66 \%). No rio Javaés, tem-se verificado a ocorrência de irregularidades no padrão dos escudos do casco em exemplares adultos e jovens de ambos os sexos para $P$. expansa e P. unifilis, semelhantes aos registrados em filhotes recém-eclodidos.

Mallmann (1994) submeteu ninhadas de jabuti, $C$. carbonaria a diferentes regimes de temperatura, obtendo um aumento da mortalidade e filhotes com diferentes padróes de escutelação (excesso ou supressão de escudos), nos escudos costais, vertebrais, marginais e femorais, além de deformaçōes na cabeça, maxila, mandíbula, ausência de olhos, má formação da cauda e anomalias na forma e disposição dos órgãos internos. A autora também comenta que além da influencia da temperatura de incubação, algumas dessas anomalias podem ter sido decorrentes de problemas genéticos dos embriōes ou devido ao manuseio.

Andrade \& Abe $(1992,1993)$ avaliaram 163 filhotes de C. carbonaria apreendidos pelo IBAMA (Instituto Brasileiro de Meio Ambiente e dos Recursos Naturais Renováveis), que estavam sendo comercializados ilegalmente na região Nordeste do Brasil, e constataram que $19 \%$ deles apresentavam anormalidades no casco, algumas ocorrendo em maior número. No entanto, não registraram anomalias graves, como as descritas nos trabalhos experimentais. Este fato, segundo os autores, pode estar relacionado ao descarte realizado pelos traficantes que julgavam tais animais impróprios para o comércio ou os embriōes com tais anormalidades não chegaram a eclodir. Dessa forma concluem que a incidência de tais anormalidades na natureza pode ser ainda maior.

Alguns experimentos realizados em laboratório apontam que fatores como temperatura, umidade, potencial hídrico, e tensões gasosas dentro do ninho, podem implicar na mortalidade e surgimento de anomalias nos embriōes de répteis (Lynn \& Ullrich, 1950; Packard et al. 1987; Packard \& Packard, 1988). O ambiente hídrico e termal dos ninhos dos quelônios pode ser influenciado por vários fatores ambientais e ecológicos, dentre eles a profundidade do ninho (Burger, 1976; Wilhoft et al., 1983), posicionamento do ninho e cobertura vegetal (Vogt \& Bull, 1984) e data da desova (Mrosovsky et al., 1984).

As duas espécies estudadas apresentam diferentes tamanhos de ninhadas (em média 100 ovos para P. expansa e 20 ovos para $P$. unifilis), profundidade dos ninhos (média de $80 \mathrm{~cm}$ para $P$. expansa e $30 \mathrm{~cm}$ para $P$. unifilis) e tempo de incubação dos ovos (média de 60 dias para $P$. expansa e de 70 dias para P. unifilis) (Pritchard \& Trebbau, 1984; Ernst \& Barbour, 1989; IBAMA, 1989a, 1989b; Fachín-Terán, 1993). A quantidade e tipo de anomalias encontradas entre $P$. expansa e P. unifilis, possivelmente estão relacionadas a algumas de suas características biológicas e comportamentais. 
No rio Javaés, a distribuição dos ninhos de $P$. expansa e P. unifilis mostra uma clara preferência desses quelônios por ambientes sedimentológicos diferenciados, seja em relação à morfologia, constituição granulométrica ou altura dos ninhos em relação ao nível do rio. Os ninhos de $P$. expansa concentram-se na porção central das praias com uma altura superior a 3,3 m em relação ao nível do rio, já os de $P$. unifilis, distribuem-se, preferencialmente, nas porções montante e jusante das praias onde os depósitos arenosos raramente ultrapassam $1,5 \mathrm{~m}$ de altura no momento da desova (Ferreira Jr., 2003; Ferreira Jr. \& Castro, 2004). Nas praias estudadas, a distribuição dos ninhos dessas duas espécies pode resultar em diferentes níveis de umidade e temperatura entre eles e, assim, gerar uma variação no índice de mortalidade e de incidência de anomalias nos embriōes de $P$. expansa e $P$. unifilis.

Segundo Packard et al. (1982) os ovos de casca rígida apresentam um pequeno potencial de trocas hídricas e gasosas com o meio enquanto que os de casca flexível, com uma camada calcária porosa, são mais dependentes do ambiente hídrico. P. expansa apresenta um ovo esférico e com casca flexível, enquanto que os ovos de $P$. unifilis são alongados e de casca rígida (Ernst \& Barbour, 1989). No rio Javaés, encontramos uma maior incidência de defeitos em $P$. expansa $(13,32 \%)$ do que em $P$. unifilis (4,44\%) o qual, aparentemente, está associado à variação na composição das cascas dos seus ovos e do seu potencial de interação com o ambiente externo ao ninho. Neste sentido, é possível construir a hipótese de que, provavelmente, as espécies de quelônios que possuem ovos de casca flexível geram uma maior proporção de filhotes com defeitos do que as espécies com ovos de casca rígida.

A ocorrência de adição e/ou supressão de escudos no casco, apresentam-se, provavelmente, como não letais, uma vez que exemplares adultos têm sido encontrados na natureza com tais defeitos (Cagle, 1950; Lynn \& Ullrich, 1950; Molina, 1995). Mas, como ressaltam Mast \& Carr (1989), os padrôes anormais no casco representam expressões fenotípicas de algum problema morfológico ou fisiológico, ainda não manifesto, que pode afetar a viabilidade do indivíduo.

Essas diferenças na proporção de filhotes com irregularidades no casco e a localização desses defeitos entre $P$. expansa e $P$. unifilis podem ser uma resposta diferenciada não só às condições ambientais que os ninhos dessas espécies estão sujeitos, como por exemplo, a localização, a profundidade e o tempo de incubação, mas, principalmente, ao tipo de casca que constituem os ovos.

O transplante de ninhadas, que muitas vezes é realizado em áreas protegidas pelo RAN/ IBAMA, deve ser visto com cautela. Considerando que as condiçóes do novo ninho não devem diferir dos locais selecionados pelas fêmeas para postura, tendo em vista que as condiçóes ao serem diferenciadas podem ocasionar, a partir de uma incubação inadequada, o surgimento de ninhadas com alta incidência de filhotes com padrão irregular dos escudos do casco.

\section{AGRADECIMENTOS}

Este trabalho só foi possível com o apoio recebido pelo NATURATINS, através de Isac Braz da Cunha. O apoio às etapas de campo foi dado pelo RAN/IBAMA, pelo Instituto Ecológica e Earthwatch Institute. Nos gostaríamos de agradecer a Divaldo Rezende, Stephano Merlim, Mariluce Messias e Maria Tereza do Instituto Ecológica e Antônia Lúcia, Yeda Bataus e Selma Cristina do RAN/IBAMA. Agradecemos aos professores da Universidade Federal do Tocantins, Paulo Dias Ferreira Jr. e Lilyan Luizaga, da Universidade de São Paulo, Ana Maria de Souza e Érika Schlenz pela ajuda prestada. Agradecemos também aos voluntários do Earthwatch Institute que participaram ativamente da coleta de dados em 2001, 2002 e 2003.

\section{BIBLIOGRAFIA CITADA}

Andrade, D.V. \& Abe, A.S. 1992. Anomalias em neonatos de jabuti Geochelone carbonaria (Testudinata, Testudinidae). Resumo publicado nos Anais do XII Congresso Latino-Americano de Zoologia e XIX Congresso Brasileiro de Zoologia. Belém (PA), $131 \mathrm{p}$.

Andrade, D.V. \& Abe, A.S. 1993. Natural occurrence of shell abnormalities in hatchling red-footed tortoises (Geochelone carbonaria). Herpetological Review, 24 (3): 89.

Burger, J. 1976. Temperature relationships in nests of the northern diamondback terrapin, Malaclemys terrapin terrapin. Herpetologica, 32 (4): 412-418.

Cagle, F.R. 1950. The life history of the slider turtle, Pseudemys scripta troostii (Holbrook). Ecological Monographs, 20 (1): 31-54.

Ernst, C.H. \& Barbour, R.W. 1989. Turtles of the World. Washington, Smithsonian Institution Press. 313 pp.

Ewert, M.A. 1979. The embryo and its egg: development and natural history, In turtles: perspectives and research. Harless M. y H. Morlock. John Wiley and Sons. Inc. New York, p. 333-413.

Fachín-Terán, A. 1993. Caracteristicas de Podocnemis unifilis (Reptilia, Testudines) en el rio Samiria, Loreto. Boletim de Lima, 87: 69-74.

Ferreira Jr., P.D. 2003. Influência dos processos sedimentológicos e geomorfológicos na escolha das áreas de nidificação de Podocnemis expansa (tartaruga-da-amazônia) e Podocnemis unifilis (tracajá), na bacia do rio Araguaia. Ouro Preto, Universidade Federal de Ouro Preto. (Tese de Doutorado em Ciências Naturais, Universidade Federal de Ouro Preto). 296 pp.

Ferreira Jr., P.D. \& Castro, P.T.A. 2004. Geological control of Podocnemis expansa and Podocnemis unifilis nesting areas in rio Javaés, Bananal Island, Brazil. Acta Amazonica, 33 (3): 445 468. 
IBAMA - Instituto Brasileiro do Meio Ambiente e dos Recursos Naturais Renováveis. 1989a. Projeto Quelônios da Amazônia - 10 anos. IBAMA, 119 pp.

IBAMA - Instituto Brasileiro do Meio Ambiente e dos Recursos Naturais Renováveis. 1989b. Projeto Quelônios da Amazônia: Manual Técnico/IBAMA. IBAMA, 125 pp.

Lynn, G. \& Ullrich, S.M.C. 1950. Experimental production of shell abnormalities. In turtles. Copeia, (4): 253-262.

Mallmann, M.T.O. 1994. Influência da temperatura de incubação na determinação sexual em Geochelone carbonaria (Spix, 1824) (Reptilia, Testudines, Testudinidae). Porto Alegre, Pontifícia Universidade Católica do Rio Grande do Sul. (Dissertação de Mestrado em Zoologia, Pontifícia Universidade Católica do Rio Grande do Sul). 52 pp.

Malvasio, A. 2001. Aspectos do mecanismo alimentar e da biologia reprodutiva em Podocnemis expansa (Schweigger,1812), Podocnemis unifilis (Troschel, 1848) e P. sextuberculata (Cornalia, 1849) (Testudines, Pelomedusidae). São Paulo, Universidade de São Paulo. (Tese de Doutorado em Zoologia, Instituto de Biociências da Universidade de São Paulo). 199 pp.

Mast, R.B. \& Carr, J.L. 1989. Carapacial scute variation in Kemp's Ridley sea turtle (Lepidochelys Kempi) hatchlings and juveniles. In: International Symposium on Kemp's Ridley Sea Turtle Biology, Conservation and Management. National Marine Fisheries Service Southeast, p. 2002-2219.

Medem, F. 1969. Estudios adicionales sobre los Crocodylia y e Testudinata del Alto Caquetá y río Caguán. Caldasia, 10 (48): 329-353.

Medem, F. 1976. Recomendaciones respecto a contar el escamado y tomar las dimensiones de nidos, huevos y ejemplares de los Crocodylia e Testudines. Lozania, 20: 1-17.

Molina, F.B. 1989. Observações sobre a biologia e o comportamento de Phrynops geoffroanus (Schweigger, 1812) em cativeiro (Reptilia, Testudines, Chelidae). São Paulo, Universidade de São Paulo. (Dissertação de Mestrado em Zoologia, Instituto de Biociências da Universidade de São Paulo). 185 pp.

Molina, F.B. 1992. O comportamento reprodutivo de quelônios. Biotemas, 5: 61-70.

Molina, F.B. 1995. Observaçóes sobre a biologia e o comportamento reprodutivo de Trachemys dorbignyi (Duméril \& Bibron, 1835) em cativeiro (Reptilia, Testudines, Emydidae). São Paulo,
Universidade de São Paulo. (Tese de Doutorado em Zoologia - Instituto de Biociências da Universidade de São Paulo). 307 pp.

Molina, F.B \& Rocha, M.B. 1996. Identificação, caracterização e distribuição dos quelônios da Amazônia brasileira. Apostila da aula ministrada no mini-curso "Metodologia de Pesquisa e Classificação de Quelônios”, realizado durante o "XI Encontro sobre Quelônios da Amazônia“, organizado em agosto de 1996, em Belém (PA), pelo CENAQUA/IBAMA.

Mrosovsky, N.; Hoppkins-Murphy, S.R. \& Richardson, J.I. 1984. Sex ratio of sea turtles: seasonal changes. Science, 225: 739740.

Packard, M.J.; Packard, G.C. \& Boardman, T.J. 1982. Structure of eggshells and water relations of reptilian eggs. Herpetologica, 38 (1): 136-155.

Packard, G.C., Packard, M.J., Miller, K. \& Boardman, T. J. 1987. Influence of moisture, temperature, and substrate on snapping turtle eggs and embryos. Ecology, 68 (4): 983-993.

Packard, G.C. \& Packard, M..J. 1988. The physiological of reptilian eggs and embryos. In: Biology of the reptilia. Ed. Gans and Huey. New York (USA), p. 523-605.

Pough, F.H.; Heiser, J.B. \& McFarland, W.N., 1993. A Vida dos Vertebrados. Atheneu Editora. São Paulo (SP), 839 pp.

Pritchard, P.C.H. 1979. Encyclopedia of Turtles. T.F.H. Publ. Inc., Neptune, New Jersey. 859 pp.

Pritchard, P.C.H. \& Trebbau, P. 1984. The Turtles of Venezuela. Society for the study of amphibians and reptiles. $403 \mathrm{pp}$.

Storer, T.I.; Usinger, R.L.; Stebbins, R.C. \& Nybakken, J.W. 1989. Zoologia Geral. Sexta Edição Revista e Aumentada (Segunda Reimpressão). Companhia Editora Nacional. 816 pp.

Vogt, R.C. \& Bull, J. 1984. Ecology of hatchling sex ratio in map turtle. Ecology, 65 (2): 582-587.

Wilhoft, D.C.; Hotaling, E. \& Franks, P. 1983. Effects of temperature on sex determination in embryos of the snapping turtles, Chelydra serpentina. Journal of Herpetology, 17 (1): 38-42.

Zangerl, R. \& Johnson, R.G. 1957. The nature of shield abnormalities in the turtle shell. Fieldiana Geol., 10 (29): 341-362.

Recebido em 27/11/2007

Aceito em 18/11/2008 